\title{
Imaginar un hogar: desplazamiento psicológico y su relación con la imaginación en tres obras de Gloria Anzaldúa
}

Resumen: Este ensayo presenta a la autora Gloria Anzaldúa y su género innovador de autohistoria para trazar la trayectoria de lo queer como desplazamiento psicológico en tres de sus historias "La Prieta”, "El Paisano is a Bird of Good Omen” y "Reading LP”. Su personaje autobiográfico se enfrenta con realidades distintas en cada autohistoria, lo cual permite a Anzaldúa explorar la sociedad en la que vive e imaginar alternativas más hospitalarias para la gente queer. El desplazamiento que acompaña a los personajes queer es un trauma que causa dolor por ser otreado por la sociedad. La autora plantea una visión que denomina el Mundo Zurdo, un refugio en el que los queers de todo tipo pueden unirse para construir un nuevo mundo en el que las diferencias no se borran, sino que se celebran. Tanto Anzaldúa, como sus personajes autobiográficos sufren constantemente por sentirse desplazados a pesar de no haber salido del país, una experiencia que influenció considerablemente el desarrollo de su vida y su teorización de lo queer. Anzaldúa propone utilizar este dolor como catalizador para superar el sufrimiento de sentirse desplazada y llega a establecer la imaginación como sitio material con la potencialidad de cambiar el mundo.

Palabras clave: Anzaldúa, desplazamiento, dolor, queer, otrear

El desplazamiento tiene consecuencias en el aparato emocional, independientemente de si se trata de un movimiento migratorio o dentro de un mismo país, ya que ser otreado dentro de la propia cultura causa dolor emocional en forma de trauma. En este artículo se exploran las ramificaciones emocionales de las identidades interseccionales de la escritora texana Gloria Anzaldúa y de sus protagonistas autobiográficas en tres autohistorias. "La Prieta" fue publicado en la pionera antología This Bridge Called My Back: Writings by Radical Women of

Quisiera agradecer a Danae Gallo González por la invitación a contribuir al libro y por su revisión y traducción de este ensayo.

Betsy Dahms, University of West Georgia 
Color que Anzaldúa co-editó con Cherríe Moraga en 1981. El segundo ensayo "El Paisano is a Bird of Good Omen” se publicó en Conditions en 1982 y el último, “Reading LP” vio la luz póstumamente en Gloria Anzaldúa Reader, de 2009.

Anzaldúa habla del sentido de ser del otro lado en varios aspectos de su identidad, tanto en su género, clase, "raza” y orientación sexual, como en su orientación espiritual. Este desplazamiento psicológico, o sea el trauma de ser otreado, se manifiesta en cada historia con fines diferentes para esta investigación. Además de examinar las maneras en las que se siente alienada de su familia, "raza", género y ser, Anzaldúa analiza la sensación de pertenencia con el concepto de hogar en sus obras y denomina "terrorismo íntimo" al dolor de existir entre varias “tierras”. Este terrorismo es la violencia que sentía al vivir como chicana en la frontera de Estados Unidos y México, de ser mujer en el patriarcado, de ser mujer queer en una matriz de heteronormatividad y ser intelectual con raíces campesinas en la academia. En particular, este capítulo se centra en la idea de desplazamiento interno, como dice Anzaldúa, “[a]lienated from her mother culture, 'alien' in the dominant culture, the woman of color does not feel safe within the inner life of her Self” (Anzaldúa 1999: 42). Es este sentido profundo de vivir separada, sin hogar, ni seguridad, el que suelen leer los lectores sin percatarse de la importancia de la declaración, quizás por privilegio racial o sexual. Así que si Anzaldúa -mujer de color, chicana y queer en Estados Unidos- no se sentía cómoda en su propio cuerpo, ¿cómo consiguió navegar por el mundo?

Primero, presentaré a la autora chicana. Luego analizaré su creación de un género nuevo, la autohistoria, que combina la autobiografía y la ficción para contar su propia historia y para expresar la realidad de muchas mujeres de color. Hablaré del poder de la imaginación para afectar la realidad en maneras tangibles con la idea del Mundo Zurdo y finalmente, voy a trazar la trayectoria de su obra a través de tres autohistorias. La protagonista de la autohistoria "La Prieta" sirve como una especie de herramienta de diagnóstico de su realidad. Es una evaluación de la sociedad contemporánea. La protagonista hace un balance de cómo funciona el mundo y cómo hay partes de ella a las que se les permite existir y otras a las que se les exilia. Andrea, la protagonista de "El Paisano is a Bird of Good Omen" se niega a conformarse con su realidad heteronormativa y homofóbica. Debido a su conexión espiritual con la naturaleza y sus

\footnotetext{
1 El Mundo Zurdo, según la definición de AnaLouise Keating, “indicates communities based on commonalities, visionary locations where people from diverse backgrounds with diverse needs and concerns coexist and work together to bring about revolutionary change" (Keating 2009: 322). "El Mundo Surdo [sic]" también es el título de otra serie de lecturas y de talleres de escritura guiados por Anzaldúa en los setenta. La $S$ chicana se sustituyó por la $Z$ con la publicación de This Bridge Called My Back sin el consentimiento de la autora.
} 
poderes sobrenaturales con los animales, es poco probable que encuentre un nicho en la sociedad en el que se le acepte y se le anime a ser como es. Con la última autohistoria, "Reading LP”, la protagonista autobiográfica dedica tiempo y energía a la construcción de una nueva realidad en vez de gastar recursos para borrar de la memoria la programación social limitante al ser. Esta nueva realidad anzalduense lleva la potencia de diferenciarse del statu quo. Es una visión de una realidad que todavía no existe, pero si existiera, cambiaría el mundo para gente otreada como Anzaldúa y sus personajes autobiográficos. "Reading LP” sugiere que cuanto antes una protagonista encuentre "hogar" en el yo, en su propia piel queer, brown, chicana, más pronto podrá recrear la realidad de forma diferente. Se puede decir que Anzaldúa y, por ende, sus personajes autobiográficos, sufrieron por el dolor del desplazamiento sin salir de su país y del seno de su familia; que la condición de su ser estuvo marcada por el desplazamiento permanente, lo que tuvo consecuencias importantes para su vida y su teorización de lo queer. Anzaldúa utiliza este dolor como catalizador para superar su situación. En efecto, llega a valorar la imaginación como sitio material con la posibilidad de cambiar el mundo. Anzaldúa plantea el Mundo Zurdo como el punto de llegada para sus personajes; puede que todos sean demasiado queer para cualquier sociedad existente. El Mundo Zurdo es el refugio en el que los queers de todo tipo pueden unirse para construir un nuevo mundo en el que las diferencias no se borran, sino que se celebran.

\section{Introducción a la autora Gloria E. Anzaldúa}

A Gloria E. Anzaldúa (1942-2004), como escritora paradigmática, se le cita muy a menudo en obras feministas estadounidenses. No obstante, no ha llegado a entenderse gran parte de su obra en su complejidad y esta sigue, además, según Linda Martin Alcoff, "undertheorized” (Martin Alcoff 2006: 256). Un recorrido por la literatura y la crítica de la literatura chicana desde los años 1980 hasta el presente revela un consistente respaldo a su innovadora obra: su antología coeditada de 1981 This Bridge Called My Back: Writings by Radical Women of Color y Borderlands / La Frontera: The New Mestiza considerada mayoritariamente un texto canónico, aparece en innumerables programas de estudio de cursos universitarios en Estados Unidos y ha contribuido a definir el entonces nuevo campo de los Estudios Fronterizos. El trabajo de Anzaldúa no solo está subteorizado en cuanto al ámbito de sus proyectos de escritura, ya que la mayoría de los estudiosos se contentan en centrarse únicamente en Borderlands. Apenas se presta atención académica a sus numerosas obras escritas antes y 
después de Borderlands, y cuando se abordan, los estudiosos retoman argumentos previamente articulados sobre la nueva conciencia mestiza. Sin embargo, la nueva conciencia mestiza, como muchas otras teorías de Anzaldúa, echó raíces antes de la publicación de Borderlands y continuó desarrollándose después. El objetivo del presente análisis es examinar sus escritos pre- y post- Borderlands en un esfuerzo por trazar la trayectoria del pensamiento anzalduense, específicamente en lo que se refiere al desarrollo de su personaje autobiográfico, que lleva el nombre de La Prieta, y a las concepciones de pertenencia y de dolor del desplazamiento.

Como se relata en Borderlands, Gloria Anzaldúa nació en el Valle del Bajo Río Grande al sur de Texas. La proximidad geográfica de la frontera entre México y Estados Unidos figura de manera prominente en su trabajo, así como su experiencia de primera mano como campesina. Las dolencias físicas de la autora también se encuentran en sus escritos: Anzaldúa revela que sufrió un desequilibrio hormonal que le provocó la menstruación a los tres meses de edad y la pubertad a los siete años, condición que la llevó a una histerectomía en 1980, a la edad de treinta y ocho años. Anzaldúa discute estos eventos en sus escritos porque la relación física con su cuerpo emerge como esencial para el desarrollo de su perspectiva sobre la escritura, la espiritualidad, la autopercepción y sus ideas sobre el conocimiento. Todas estas experiencias e ideas marcaron de manera fundamental a la autora, que se sentía desplazada sin salir de casa. En su propia familia se sentía otra y este desplazamiento se manifiesta en lo físico, lo emocional y lo metafísico en su vida y en sus obras publicadas.

Borderlands / La Frontera: The New Mestiza combina varios géneros y perspectivas para ofrecer una intervención racial y sexual al movimiento feminista de esta época que, desafortunadamente, por los pocos avances en los estudios a su obra, es todavía relevante hoy en día. Su teorización de los borderlands o zonas fronterizas entre países, entre culturas, entre personas o estos mismos límites dentro de una sola persona provoca un estudio fructífero y hasta un campo de estudio entero. Concentrarse en lo del medio, en el espacio fronterizo, o lo que Anzaldúa más tarde en su carrera llamaría nepantla y que otros han tachado de lo liminal, abre un campo fructífero de investigación tanto en su sentido geográfico como en lo personal. ${ }^{2}$

2 Anzaldúa alude a lo liminal en términos antropológicos con los estudios de Lévi-Strauss. Otra autora indígena, Paula Gunn Allen, invoca la liminalidad en su poesía, en la que trata de la "raza" y la homosexualidad, diciendo que la homosexualidad es una condición liminal que acompaña al sujeto toda la vida. Keating cita una entrevista de 1993, donde Gunn Allen "associates her lesbianism with the Native American worldview and explains that 'perversity (transformationality) 


\section{La creación de un género literario nuevo: la autohistoria}

A Anzaldúa se le conoce sobre todo por su obra Borderlands y por la hibridez que le caracteriza. Este libro está escrito en una mezcla de inglés estándar y dialectos de la clase trabajadora, español estándar, mexicano, mexicano-norteño, español chicano, tex-mex y pachuco y combina la poesía con la autobiografía y referencias antropológicas (cf. Anzaldúa 1987: 55). Con esta hibridación formal en la presentación del libro se teoriza la creación de la nueva subjetividad mestiza y la nueva conciencia mestiza que especula con una teorización del espacio liminal para desmantelar el pensamiento dualista. Esto, a su vez, propone una negociación de la identidad en su entendimiento relacional y fluido. La nueva conciencia mestiza, tal como la propone Anzaldúa en Borderlands, es una llamada radical a la revolución para poner en jaque al statu quo (Anzaldua 1987: 91). Esta revolución es parcialmente promulgada a través de la re-escritura de mitos indígenas (La Llorona, Coatlicue, Coyolxauhqui) desde una perspectiva feminista para validar la experiencia vivida por Anzaldúa como chicana queer en una sociedad patriarcal. Es importante destacar que, en su exploración de la subjetividad, Anzaldúa no celebra la hibridación postmoderna. Más bien, la autora detalla el dolor de la condición muy real de vivir como sujeto híbrido, al mismo tiempo que ofrece, con la nueva conciencia mestiza, una perspectiva única al sujeto híbrido.

Una de las contribuciones teóricas de Anzaldúa es su insistencia en que el sujeto híbrido saque provecho de lo que le hace diferente y utilice esa diferenciación para empoderarse. En definitiva, Anzaldúa propone que el sujeto híbrido encuentre la fuerza en su diferencia. Lejos de abogar por una posición de asimilación, Anzaldúa desafía el statu quo para incluir un nuevo sujeto híbrido empoderado.

Anzaldúa inventa el género de la autohistoria como vehículo de esta autoreflexión y teorización. En su glosario, Keating define las autohistorias de la siguiente manera:

Anzaldúa coined this term, as well as the term autohistorias-teoría, to describe women-ofcolor interventions into and transformations of traditional western autobiographical forms. Deeply infused with the search for personal and cultural meaning, or what Anzaldúa describes in her post-Borderlands writings as 'putting Coyolxauhqui together,' both autohistoria and autohistoria-teoría are informed by reflective self-awareness employed in the service of social-justice work. Autohistoria focuses on the personal life story, but as the autohistoria tells her own life story, she simultaneously tells the life stories of others. (Keating 2009: 319)

constitutes the sacred moment, the process of changing from one condition to another-life-long liminality”' (Keating 1993: 78). 
Los tres relatos que se investigan en este capítulo son todos autohistorias que fusionan la experiencia vivida por Anzaldúa con sus personajes ficticios, que por ello se llamarán en lo sucesivo personajes autobiográficos.

\section{Imaginación material: El Mundo Zurdo}

Como persona otreada, Anzaldúa tuvo la necesidad de crear un género literario nuevo, la autohistoria, para expresar fielmente su experiencia. Además, el no encajar en la realidad que conocía, llevó a la autora a explorar las muchas maneras de ser del otro lado y a teorizar la alienación. Esta la describe en la multiplicidad de la otredad que la conduce hacia su proyecto queer de reflexionar sobre este mundo y crear mundos nuevos en sus autohistorias, algo semejante a lo que propusiera José Esteban Muñoz con la idea de “queer world making” (Muñoz 1999: 23).

Anzaldúa usaba su imaginación para crear un mundo acogedor para sus muchas formas de ser queer. La autora llama tanto al acto de crear mundos nuevos a través de la imaginación, como a la realidad imaginada con el concepto del Mundo Zurdo y lo describe como una manera visionaria de relacionarse, basándose en afinidades en vez de en semejanzas. El Mundo Zurdo es una visión queer de un mundo de justicia social colectiva.

En la obra de Anzaldúa, el Mundo Zurdo es un camino metafísico que se centra en un movimiento bidireccional: "a going deep into the self and an expanding out into the world, a simultaneous recreation of the self and a reconstruction of society" (Anzaldúa 1981: 208). Según la autora, los cambios en el mundo son posteriores a los cambios en uno mismo. Este es el aspecto visionario del pragmatismo de Anzaldúa. El Mundo Zurdo "has opened up the possibility that I may live in the world of my vision, a sort of mundo surdo, a left-handed world where creativity, intuition, the feminine will manifest and transform the planet" (Anzaldúa s.a.: Box 44, folder 19). No obstante, a través de su personaje autobiográfico, Anzaldúa admite que se siente "confused as to how to accomplish this" visión del Mundo Zurdo (Anzaldúa 1981: 207-208). Explorando un paisaje amplio de alienación en términos de "vivir sin hogar", el cuento de Anzaldúa "Reading LP" se centra en dar ejemplos de cómo regresar a casa, o sea, regresar al cuerpo o al yo, y en la importancia de la imaginación como uno de los pocos espacios en los cuales es posible vivir una realidad diferente.

Sin embargo, la teorización de la imaginación como sitio generativo no es escapista ni utópica. De hecho, Anzaldúa llega a valorar la imaginación como sitio material, como una imaginación tangible con el poder de efectuar cambios en nuestra realidad, con la posibilidad de cambiar el mundo. A este mundo 
imaginado por ella puede pertenecer siendo ella misma sin la necesidad de tener que ocultar ninguna parte de su identidad. Así crea el mundo en el que quiere y puede sobrevivir y florecer. La visión de este hogar se realiza primero en la imaginación, parecido a como lo plantea Adrienne Maree Brown en su teoría de la estrategia emergente (2017), y luego se vuelve material en esta realidad. Con este término Brown señala que las estrategias que necesita la gente marginalizada no vienen de algo ya conocido, sino que provienen de la imaginación de las mismas personas que sufren discriminación (cf. Brown 2017: 34). En efecto, Brown aboga por el poder de la materialización de la imaginación en concebir nuevas ideas. Se necesitan ideas nuevas para crear realidades nuevas porque las ideas ya existentes no han podido liberar a la gente marginalizada de la discriminación. Anzaldúa, al igual que la escritora afro-americana Octavia Butler, a quien Brown estudiaba, se instaló en lo que muchos llaman un mundo de ciencia-ficción para imaginar realidades alternativas. Pero, en contraste con Octavia Butler, Anzaldúa no lo concibió como ciencia-ficción. Para Anzaldúa, la imaginación sí tiene la posibilidad de crear un mundo material nuevo. Al presentar la imaginación como vehículo de la materialización, Anzaldúa la utiliza como una estrategia para concebir algo diferente, una realidad nueva. Toda innovación surge en primera instancia como pensamiento o imaginación.

\section{4 "La Prieta"}

En una de sus primeras publicaciones, “La Prieta”, Anzaldúa utiliza el término “QUEER” para describir su experiencia vital (Anzaldúa 1981: 199 mayúsculas en el original). Conner, Hatfield y Sparks afirman:

For Anzaldúa, the term 'queer' embraces not only lesbians, gay men, bisexuals and transgendered persons, but also perceived as different, or who self-identify as be different from, 'the norm.' It should be noted here that she first used the term 'queer' in this way in or around 1976, developing the definition in the early 1980s though she is seldom recognized as an early user of the term as it is currently employed. Also not recognized is that her use of the term embraced rather than rejected the realm of the spirit. (Conner / Hatfield / Sparks 1997: 63-64)

Las connotaciones que tiene el término en este caso concuerdan más con las primeras definiciones del término en diccionarios generalistas como:

1 a: worthless, counterfeit, b: questionable, suspicious; 2 a: differing in some odd way from what is usual or normal, b (1): eccentric, unconventional, (2): mildly insane, c: absorbed or interested to an extreme or unreasonable degree; d (1) often disparaging: homosexual (2) sometimes offensive, (3): not quite well. (Merriam-Webster Dictionary 2011) 
El término queer también se usaba de manera coloquial en la comunidad chicana de clase trabajadora en la que creció Anzaldúa junto con "las otras", "marimachos, jotitas, jotas, tortilleras", términos con los que se describía a mujeres que deseaban a otras mujeres (Reuman / Anzaldúa 2000: 35-36).

En base al contenido de "La Prieta", puede afirmarse que Anzaldúa estaba explorando en su obra todas estas definiciones de queer y cómo tanto ella como otros aplicaron este término a su vida. El título del cuento "La Prieta", que literalmente significa "la de piel oscura", impone de manera casi inmediata incluir la "raza", además del género, en la discusión sobre las contribuciones de Anzaldúa al desplazamiento interno. En este caso, la protagonista autobiográfica se ve marcada como otra al nacer: "When I was born, Mamágrande Locha inspected my buttocks looking for the dark blotch, the sign of indio, or worse, mulatto blood" (Anzaldúa 1981: 198). La Prieta fue otreada racialmente por ser más oscura que su familia “de color”. Por ese motivo, se le inculcó que no se fuera "out in the sun" (Anzaldúa 1981: 198). De este modo, podría evitar que la percibieran como india siguiendo la creencia y la construcción social de que el color de piel oscura es inferior. La etiqueta "prieta", empleada por la abuela de Anzaldúa, funciona como una categoría de control sobre la joven. Anzaldúa resume el poder de las etiquetas de la siguiente manera:

Society puts us into neat little compartments to label us and keep us in our places. India, mestiza, naca, vieja, Latin spitfire, Chicana-dyke-feminist, tortillera, sexy, burro, maid, stupid, prieta, hocicona, bocona, cabrona, loser, whore, malinche, bad writer, worse thinker, undeserving, affirmative-action hire--you name it, we've heard it. (Hurtado 2011: 55)

Cada etiqueta aquí incluida ha servido para controlar a las mujeres chicanas. Según Anzaldúa, estos términos crean un desplazamiento interno en la chicana. Por su parte, la sociedad chicana utiliza estos términos para distanciar a las mujeres de sus poderes para mantenerlas dóciles y obedientes: educar a las mujeres de forma sexista es una forma de otrearlas de la cultura machista y otrearlas de sí mismas. Anzaldúa se considera otreada en la sociedad patriarcal chicana en la que nació, la cual favorecía a los hombres por encima de las mujeres. Este era también el caso de su madre, cuya "allegiance was and is to her male children, not to the female" (Anzaldúa 1981: 201). El racismo es otra forma de otrearse. La autora creció en el clima racial de la Texas de la década de los cuarenta que privilegiaba a los caucásicos en detrimento de los mexicanos, como quedaba ilustrado en los westerns de bolsillo que leía en su infancia.

Además, se sentía otreada, o queer, por su prematura y dolorosa menstruación. El inusual desequilibrio hormonal que le causó la menstruación a los tres meses de edad y aceleró su desarrollo físico hizo que Anzaldúa se sintiera como una extraña en su propia casa y en su propia piel. Su madre contribuyó a esta 
sensación, insistiendo en ocultar su crecimiento mamario con fajas de algodón apretadas "so that kids at school would not think them strange beside their own flat brown mole nipples” (Anzaldúa 1981: 199). El sentimiento de aislamiento frente a sus compañeras de pecho plano llevó a Anzaldúa a retirarse "into books and solitude and keep away from others” (Anzaldúa 1981: 199). Su interés en leer y en adquirir conocimiento también la diferenciaban de sus otros compañeros chicanos en el colegio.

Cada una de las ocho secciones de "La Prieta" describe otro aspecto de la otredad, o queerness de su protagonista y, por extensión, de Anzaldúa, y el subsiguiente desplazamiento de ambas de la sociedad mayoritaria. Finalmente, la muerte del amado padre de Anzaldúa al entrar esta en la pubertad "irrevocably shattered the myth that there existed a male figure to look after me... I lost my father, god, and my innocence all in one bloody blow" (Anzaldúa 1981: 200). Todo esto, sumado a la atracción sexual que sentía hacia su padre, así como los sentimientos y deseos sexuales hacia otras mujeres en una sociedad homófoba, dejó a Anzaldúa aislada. ${ }^{3}$ Como afirma en una entrevista con Keating, Anzaldúa nunca sintió que perteneciera a ningún lugar, sino "always on the other side”, “always felt like all my difference was visible” (Anzaldúa 2000: 170-171).

Debido a su menstruación temprana y a su acelerada madurez hormonal, Anzaldúa fue sometida a una histerectomía. Este momento vital es significativo, no solo porque es una de las cuatro experiencias cercanas a la muerte que Anzaldúa experimentó durante su vida, sino también porque tuvo el propósito didáctico de enseñarle cómo convertir su enfermedad en sanación. Como recuerda en la propia autohistoria: "My soul in one corner of the hospital ceiling, getting thinner and thinner telling me to clean up my shit, to release the fears and garbage from the past that are hanging me up. Strip away -all the way to the bone. Make myself utterly vulnerable” (Anzaldúa 1981: 203). Anzaldúa toma como base su cuerpo, sus emociones, como el miedo y otras experiencias personales como mujer queer de piel morena para teorizar sobre la dinámica del poder articulada en torno a la "raza”, el género, la orientación sexual y a su condición corpórea en su familia chicana y en la sociedad de Texas en la que se crió.

"La Prieta” es la primera publicación de Anzaldúa en la que la autora explora el desplazamiento. Con cada tipo de diferencia, Anzaldúa y su personaje autobiográfico La Prieta se aislaron más, sintiéndose rechazados por su familia y la socie-

3 Anzaldúa habla de estos intensos sentimientos hacia su padre a veces como atracción sexual y otras como sentimientos sensuales o espirituales. Véase la entrevista que realizó en 1983 con Christine Weiland (cf. Anzaldúa 2000: 79). 
dad. La Prieta no pertenecía a ninguna parte. Al menos no a un lugar en el que todas sus partes -todas las categorías identitarias interseccionales ${ }^{4}$ que ella habitaba- fueran bienvenidas. En cuanto a la trayectoria del desplazamiento psicológico de Anzaldúa, la autohistoria "La Prieta” sirve como una especie de herramienta de diagnóstico de su realidad narrada en primera persona. "La Prieta" hace un balance de cómo funciona el mundo y cómo hay partes de ella a las que se les permite existir y otras a las que se les exilia.

\section{La Prieta como Andrea en "El Paisano"}

"El Paisano is a Bird of Good Omen" es una corta autohistoria ambientada en la década de 1940-1950 en Texas, donde la protagonista - una mujer masculina, Andrea de la Cruz- duda sobre su decisión de casarse con su afeminado amigo Zenobio: "this marriage will save [them] from having to marry" (Anzaldúa 1983: 161). La historia se desarrolla en el rancho de la familia de Andrea, ${ }^{5}$ donde el lector es testigo de la recepción la noche antes de la boda. El paisano del título se refiere al correcaminos enjaulado que se trae a la recepción como regalo de bodas y, en muchos sentidos, este símbolo sirve de paralelismo con el conflicto interno de Andrea con respecto a las limitaciones que supone el matrimonio y las normas sociales que este conlleva. De hecho, la escena de la recepción queda en segundo plano cuando la autora explora los monólogos interiores de Andrea y su conexión con el rancho de su familia en Texas, en particular con los animales que Andrea cuida y con los que siente una profunda conexión espiritual. Por ejemplo, Andrea escucha los rumores de los vecinos porque tiene una víbora de mascota: "Fue un escándalo... Sleeping naked with la serpiente. A huge rattler... Her henna hair wild over her body, her body glistening, the serpent entwined around her middle, its head peering from her pubic patch"

4 La interseccionalidad es un término que acuñó Kimberlé Crenshaw en 1989 para describir las experiencias de mujeres afroamericanas quienes, por la intersección de "raza” y género, se enfrentan con sistemas de opresión y marginalización entrelazados. Vemos huellas teóricas con el pensamiento de Du Bois (1969), Baldwin (1963), hooks (1981) y Anzaldúa (1987), por mencionar solo algunos autores.

5 El personaje de Andrea comparte muchas similitudes con otras protagonistas anzalduenses, especialmente con la Prieta de "La Prieta" y la Prieta de la autohistoria "Reading LP", que se discute más adelante en este artículo. En una entrevista de 1991 con AnaLouise Keating, Anzaldúa, comenta al hablar de una novela en relatos inédita, titulada Prieta: The Dark One, que el personaje de Andrea en "El Paisano" se ha transformado en el personaje de Prieta (cf. Anzaldúa 1991: 161). Es decir, su recurrente protagonista Prieta se ha convertido en Andrea. 
(Anzaldúa 1983: 159). Anzaldúa discutió este sentimiento de interconexión en una entrevista con Keating en 1993 en los términos siguientes: "here is the socalled real world and there's a wall, a partition, and then over here is the world of spirit and the world of ideals. I don't think we need that partition" (Anzaldúa 2000: 115). A través de su protagonista Andrea, Anzaldúa desestabiliza la división entre lo espiritual y lo material y, de esta manera, vincula la queerness de Andrea y su espiritualidad. Así, el desplazamiento de la sociedad implica, en este caso, una conexión más profunda con el mundo natural.

$\mathrm{Al}$ igual que otros protagonistas anzalduenses, Andrea no se ajusta a la visión de su sociedad sobre la expresión de género y el deseo sexual tolerado. Antes de que el lector se entere de que Andrea prefiere usar pantalones de hombre (cf. Anzaldúa 1983: 154), tiene pelo en las axilas, hace trabajo de hombres y se fija en la chica López por sus “nice tits” (Anzaldúa 1983: 161), se explicita su conexión con la tierra: sobre un poste de mezquite del corral, el poste "seems an appendage of herself, a fifth limb, one that's also part of the corral, the corral that's part / of the land" (Anzaldúa 1983: 153). Después de esta conexión entre Andrea y el corral / la tierra, Anzaldúa incluye un símil aparentemente inocuo a través del que relaciona los postes con la gente y el corral con la sociedad: "if the tops of the posts are not flush with the average height their heads are either lopped off to make a tidy corral or they are cast out as deficient, unsuitable" (Anzaldúa 1983: 153-154). No obstante, este símil prefigura el conflicto central de la historia. Nótese la interesante elección de la palabra -heads, cabezas-, ya que no es común describir la parte superior de los postes de manera antropomórfica. Andrea, por muchas razones, es uno de los postes que no cabe; es desafiante con su madre, es misteriosa y sus vecinos la temen, y, además, se casa a regañadientes con Zenobio.

Estos dos personajes principales, Andrea y Zenobio, exhiben una expresión de género inconformista. A Andrea se le retrata masculina y a Zenobio se le asocia con comportamientos femeninos tradicionales: cocinar, coser y estar guapo (cf. Anzaldúa 1983: 169). Ambos personajes señalan la maleabilidad de las construcciones sociales de género. Muchos teóricos han contribuido a la discusión sobre la construcción social del género y de la sexualidad. Entre ellos destacan West y Zimmerman, quienes en su artículo "Doing Gender" (1987) extraen el sexo de la expresión de género. Para los autores, el género es una actuación que no podemos evitar llevar a cabo en nuestra vida cotidiana (cf. West / Zimmerman 1987: 147). Judith Butler también aplica el trabajo lingüístico de John L. Austin sobre las expresiones performativas que promulgan el acto de habla a las cuestiones de identidad y más específicamente al género. Por ejemplo, el acto de habla performativo "ahora os declaro marido y mujer" vincula legalmente a los dos referentes en el matrimonio por la auto- 
ridad otorgada al enunciado en su repetición. Butler separa el sexo del género; el sexo se basa en gran medida en los genitales y los cromosomas, mientras que el género es un comportamiento social que uno hace. Que una persona actúe de manera masculina si tiene un cuerpo masculino, hace referencia a actuaciones previas de comportamiento masculino socialmente aceptado $\mathrm{y}$, en efecto, convierte al hombre en masculino y recompensa su comportamiento de género apropiado. Butler se apresura a señalar que la actuación de género no siempre coincide con el sexo del intérprete y advierte que cualquier tipo de incongruencias de este tipo suelen ser castigadas socialmente (cf. Butler 1990: 33). La regulación de las fronteras entre el género y el sexo es precisamente lo que hace que Zenobio y Andrea tengan que casarse para evitar el castigo social.

El telón de fondo de la historia, la cena en la casa de la novia la noche antes de su boda en Texas entre los años cuarenta y los cincuenta lo crea la heterosexualidad obligatoria impuesta por la sociedad. El matrimonio es una forma de amoldarse a la norma para que ni Andrea ni Zenobio sean "cortados" para encajar en la sociedad o "cast out as deficient” (Anzaldúa 1983: 153-154). Adrienne Rich ${ }^{6}$ explicaba en 1980 en su artículo "Compulsory Heterosexuality and Lesbian Existence" cómo las sociedades heteronormativas y homófobas no dan mucho margen a los inconformistas. Analizaba también que a las personas que han crecido en estas circunstancias se les guía desde la misma infancia a seguir el camino "recto"7 y "estrecho" de la heterosexualidad y del matrimonio, independientemente de sus inclinaciones y deseos sexuales personales (cf. Rich 1980: 640). Tanto Andrea como Zenobio están participando en una representación de heterosexualidad obligatoria, pero es una representación queer, ya que ambos saben que el ritual solo les proporcionará cierta seguridad social, la de enmascarar superficialmente su condición de queer que tanto la familia como el pueblo ya han notado. Debido a la abundancia de deseo homosexual en la fiesta de la boda (se ponen en escena los encuentros sexuales de Zenobio con José Manuel, la tendencia de Don Efraín a acercarse demasiado a Zenobio y hablarle en susurros, a Andrea bailando con Belinda López), no parece desacertado discutir si la idea de Sedgwick de un armario de cristal (Sedgwick 1990) es aplicable a este caso: si bien la sociedad nunca permitirá que los personajes vivan abiertamente su inconformidad sexual, su diferencia es un secreto a voces. No obstante,

\footnotetext{
6 El trabajo de Adrienne Rich es paralelo al estudio de Sedgwick sobre los hombres en las novelas británicas, ya que Rich explora las relaciones femeninas del mismo sexo en la correspondencia epistolar del siglo XIX, lo que le lleva a armar su teoría del continuum lésbico. Por supuesto, su trabajo también difiere en varios puntos importantes. Desafortunadamente, esa discusión no entra en el alcance de este capítulo.
}

7 En inglés, 'straight' significa recto, pero también heterosexual. 
si todo el pueblo sabe que Andrea y Zenobio no son heterosexuales, ¿por qué proseguir con la boda? Para Andrea, este matrimonio asegurará su futuro en tanto en cuanto podrá quedarse con su tierra, ya que, en este momento sociohistórico, como mujer no hubiera podido heredar ninguna propiedad. Únicamente a partir de su unión con Zenobio se le permitirá heredar el territorio ancestral y podrá, de esta manera, mantener su conexión espiritual con la tierra. Para Zenobio, este matrimonio compensará su fallida expresión de género y le otorgará un estatus completo de hombre chicano.

Al principio de la historia el lector se entera de que Andrea está menstruando - "anda en la garra"- y que durante su periodo "she feels fragile, expansive, the limits of her body stretched beyond her skin, she flows out like a sheet, encompassing, covering trees, people, everything around her" (Anzaldúa 1983: 154). Su identidad y su cuerpo se representan en ambos casos como fluidos: por un lado, en sentido literal, en un abrir y cerrar de ojos es capaz de moverse desde la cima del poste de mezquite hasta el monte donde pastorea el ganado, por el otro, también en el sentido de la teoría queer de la identidad como relacional y provisional. Andrea es, además, fluida en el sentido de que tiene una conexión espiritual con la naturaleza antes señalada. Este análisis se basa en la observación de Keating de que "the protagonist in this story enacts Anzaldúa's holistic, participatory epistemology and her definition of queer, a definition that includes but goes far beyond sexual identity" (Keating 2009: 51).

El deseo de Andrea de vivir su vida según sus propias reglas le impulsa a montar toda una escena en la fiesta de la boda. Tras el tradicional baile inaugural de los novios, Andrea se acerca a Belinda López, quien ha estado "turning down man after man, all bunched up around her like cattle around a salt lick, tongues falling out” (Anzaldúa 1983: 171). Andrea comienza a bailar con Belinda en plena conexión con la música e ignorando que todos los demás han abandonado la pista de baile porque su "pelvis makes circles around Belinda's navel” (Anzaldúa 1983: 171). Cuando Belinda la insta a que se detenga, Andrea le espeta "I'm tired of all the millions of things we're not supposed to do” (Anzaldúa 1983: 171), resaltando así su frustración con el statu quo y presagiando su inminente partida. Al igual que el paisano salvaje había anhelado su libertad, Andrea se da cuenta de que ella también debe escapar de los estrechos límites de su sociedad. El lugar al que irá es incierto; solo sabe que necesita irse, “away from here” (Anzaldúa 1983: 175). Habla con Zenobio de la posibilidad de encontrar aceptación a su inconformidad de género y sexual en el mundo "gavacho [sic]" (Anzaldúa 1983: 173), pero dado el clima racial de los años cuarenta y cincuenta, parece poco probable que pudieran vivir tranquilos en un país blanco. 
Asimismo, habida cuenta de su conexión espiritual con la naturaleza y sus poderes sobrenaturales, es poco probable que encuentre un lugar en el que se le acepte y se le anime a ser como es. En última instancia, Anzaldúa podría plantear el Mundo Zurdo como punto de llegada; puede que Andrea sea demasiado queer para cualquier sociedad existente. Andrea está exiliada de la familia y de la comunidad que ha conocido por su queerness y el final abierto de la autohistoria sugiere que solo encontrará paz en un mundo ideal. En el Mundo Zurdo la conexión de Anzaldúa entre la espiritualidad y la queerness resurge con mayor evidencia. Los queers y las personas con tendencias espirituales son capaces de habitar el Mundo Zurdo como son.

\section{La Prieta como LP en "Reading LP"}

En un mundo poco hospitalario para gente como Anzaldúa por ser queer, brown, y otras formas de ser raro, dedicarse a la autoestima y al auto-cuidado es un acto revolucionario. ¿Cuánto valor requiere declarar que es el sistema el que necesita arreglo, no el yo el que no encaja en el sistema? (cf. Anzaldúa 1981: 202). Para teorizar su propia vida, Anzaldúa llama a la protagonista con su apodo de la niñez, La Prieta, pero lo emplea con una distancia narrativa que le hace posible analizar su experiencia desde la tercera persona. El acto de salir del yo para ir a ver y empatizar con otros es, según W.E.B. Dubois, una forma de doble conciencia que experimentan personas discriminadas (cf. Du Bois 1969). La doble conciencia requiere una separación del yo. El compromiso con la introspección de la autora, por ende, le invita al lector a explorar sus propios compromisos con su ser en cuanto al amor y el cuidado propios. Paradójicamente, el compromiso con el yo implica el valor de entregarse a algo más allá del yo. Anzaldúa escribe:

I spent the first half of my life learning to rule myself, to grow a will, and now at midlife, I find that autonomy is a boulder on my path that I keep crashing into... I have always been aware that there is a greater power than the conscious I. That power is my inner self, the entity that is the sum total of all my reincarnations, the godwoman in me. (Anzaldúa 1999: 72)

Al depender de su riqueza interna y de la imaginación de pensar otra realidad posible, la autora se hizo de nuevo. En el prefacio de un volumen colectivo, la autora comentaba:

Imagination, a function of the soul, has the capacity to extend us beyond the confines of our skin, situation, and condition so that we can choose our responses. It enables us to reimagine our lives, rewrite the self, and create guiding myths for our times. (Anzaldúa 2002: 5) 
En esta cita se observa la importancia que la autora le otorga al hecho de entregarse a la imaginación, como lo hace en la autohistoria "Reading LP", donde Anzaldúa utiliza su personaje autobiográfico, La Prieta, pero ahora solo con las iniciales -LP-, quien es quizás, una versión de más edad, con más experiencia en la vida. Anzaldúa escribe: "Prieta experiences a sense of self that is and is not tied to time, space or society... She undergoes a radical shift in the way of seeing the world, a coming-to-consciousness results in changes in her identity" (Anzaldúa 2009: 250). Así señala una nueva forma de ser para su protagonista autobiográfica.

Keating publica la autohistoria "Reading LP" en la colección The Gloria Anzaldúa Reader (2009) después de la muerte de la autora. Como cuento, "Reading LP” recorre más de veinticuatro páginas, es menos pulido que sus otras obras publicadas y se distingue por la caracterización de la protagonista. LP es un personaje femenino fuerte y queer que no sufre de alienación social, ni de su sentido de ser queer, ni de la tierra heredada de la familia. Esta caracterización se distingue de las otras versiones de La Prieta que Anzaldúa había escrito a través de los años. La versión anterior de La Prieta con el nombre de Andrea en "El Paisano is a Bird of Good Omen" es tan femenina y queer como LP, pero, a diferencia de esta, sufre de la discriminación externa de su ser y acaba por dejar a su familia, su tierra y su cultura al final del cuento. LP, por su parte, no lucha con la encarnación propia, pero tampoco tiene contacto con su familia. Trabaja "como hombre" en el rancho familiar que ella renombrara "La Tigra" el nombre original era "El Tigre"- para corresponderle en su género y como un esfuerzo de LP por recuperar lo femenino. Además, LP se encarga del trabajo en el rancho, baila en el campo santo de sus antepasados después de comer el almuerzo y come tortillas integrales que su novia no considera comida auténtica Tex-Mex. Y mientras reside en muchos puestos liminales, como mujer ocupándose de trabajos tradicionalmente masculinos, como mujer queer en una sociedad homófoba y como ranchera que estudia literatura en la universidad, está comprometida con la autosuperación. Hasta que un libro que está leyendo para la universidad, que se lo presta su primo queer, le sirve a la protagonista como actante para saltar las fronteras entre las realidades. En vez de un título, el libro lleva un glifo misterioso que significa “entremados” (Anzaldúa 2009: 253). "Entremados” para Anzaldúa se refiere al espacio entre paredes, o sea, entre las maderas de la pared. Para resumir el cuento, el libro misterioso le lleva a una acequia, le hace imaginar una fogata donde quema papel y una lluvia que le hace perder su cosecha.

Siniestramente, el contenido del libro cambia de repente para reflejar la realidad de LP. Es el mismo libro que usa para una de sus clases de literatura en la universidad. En su compulsión por leer el libro, que le lleva a desplazarse 
en la percepción, pierde tiempo precioso con su amada, quien tiene muchas responsabilidades familiares y muy poco tiempo para LP. El libro la consume o, mejor dicho, LP se entrega al poder misterioso del libro. Cada vez que lee el texto, LP se sumerge en el poder de la ficción para borrar las fronteras entre realidades. En clave de lo real maravilloso se ve su mano traspasar físicamente el poste de cama. Entonces LP se cae y se encuentra en la recámara de su novia en el otro lado del pueblo. Más tarde, de nuevo en su propia casa, la protagonista observa las diferencias en los marcos de las puertas en su casa, cómo se superponen y empiezan a multiplicarse, mientras piensa: “I don't know, maybe something is missing in my life. All I do is work from before sunup to after sundown. Maybe what's missing is on the other side" (Anzaldúa 2009: 269). Llegado a este punto Anzaldúa presenta la multiplicidad de realidades como una forma de lo queer. Se trata de una protagonista queer pasando a otras realidades con la ayuda de un libro misterioso que le prestó su primo queer. El cuento termina con la promesa de LP de desmontar las paredes que la confinan; sin estas fronteras no se sentirá como si viviera "entremados", o entre paredes, el espacio incómodo de nepantla. Libre de las expectativas sociales, desmontando marcos de puerta o limitaciones sociales, puede vivir una vida más completa. Anzaldúa deja a los lectores con la promesa de que LP intentará viajar el día siguiente al otro lado, o sea, a una realidad nueva.

A diferencia del yo autobiográfico en "La Prieta", el yo paralizado en Borderlands / La Frontera: The New Mestiza o la protagonista Andrea, forzada a dejar su tierra natal, LP se entrega al libro misterioso y está empoderada a hacer los cambios que imagina; los cambios la llevan a la promesa de crear un mundo diferente. No se sabe al final del cuento si la novia le acompañará a este hogar reconstruido, pero el cuento supone una contribución optimista a la visión del Mundo Zurdo. En esta visión se dedica tiempo y energía a la construcción de una nueva realidad en vez de gastar recursos para borrar de la memoria la programación social limitante al ser y al desarrollo personal. Esta nueva realidad del Mundo Zurdo contiene la potencia de la diferencia del statu quo de la realidad material. "Reading LP" sugiere que cuanto más pronto una protagonista encuentre "hogar" en el yo, en su propia piel queer, brown, chicana, antes podrá recrear la realidad de forma diferente. Para este acto de recreación, por cierto, se requiere valor para excavarse y voluntad de entregarse a algo más allá de uno mismo. Es una tarea exigente y difícil. 


\section{Conclusión}

La gente queer puede sentirse migrante en su propio país, incluso en su propio cuerpo. La discriminación racial, de clase, de género y de orientación sexual puede causar un exilio interno a causa de la separación de la cultura dominante y hasta la separación del ser dentro de uno mismo. La disociación corporal es un resultado posible de la discriminación. El análisis aquí presentado de la caracterización del personaje La Prieta a través de tres obras de Gloria Anzaldúa sugiere que el regreso al cuerpo y la capacidad de imaginar otras realidades pueden llevar a la protagonista a la encarnación y, de allí, a crear un mundo nuevo o una nueva realidad. Es importante explicitar que Anzaldúa escribió sobre el Mundo Zurdo como concepto en sus escrituras tempranas y siguió desarrollándolo como teoría a través de su carrera. Asimismo, Anzaldúa regresaba a su personaje autobiográfico una y otra vez para imaginarlo en situaciones diferentes y en etapas distintas de su vida. La joven Prieta en "La Prieta” hace un diagnóstico del mundo y da nombre a los sistemas entrelazados de opresión. Andrea parece conformarse a la sociedad con el matrimonio de apariencias para mantener su conexión con la tierra. No es hasta LP que Anzaldúa presenta una versión de su personaje autobiográfico listo para imaginar una nueva realidad. No podemos saber por qué Anzaldúa no publicó autohistorias con LP en su vida, pero gracias a The Gloria Anzaldúa Reader tenemos un personaje listo para afrontar el reto de imaginar una nueva realidad que deja al lector con la promesa de un futuro diferente. Es interesante remarcar que "Reading LP" se escribió en 1970, antes de las otras autohistorias, pero, sin embargo, fue la última en ser publicada. No se puede saber por qué la autora no la publicó antes.

En este artículo se ha mostrado cómo Anzaldúa nos trasmite a través de sus personajes autobiográficos el dolor que le provocó sentirse desplazada sin tener que salir ni de su país, ni del seno de su familia. Este desplazamiento permanente marcó la condición de su ser y tuvo consecuencias importantes para su vida y su teorización de lo queer. A través de su obra, se puede delinear el dolor causado por este desplazamiento y su deseo, tanto de aprender de este sufrimiento, como su deseo de no sufrir. Se puede argüir que en su desesperanza y en su astucia, Anzaldúa utiliza este dolor como catalizador para superarlo. En efecto, llega a valorar la imaginación como sitio de forma material con la posibilidad de cambiar el mundo. Una imaginación material que la transporta hacia una visión que en su obra llama el Mundo Zurdo. Siente que en este mundo que ella misma ha imaginado puede ser como es, sin la necesidad de tener que ocultar ninguna parte de su identidad. Así crea el mundo al que siente que pertenece, y en el que, además, quiere y puede sobrevivir, sentirse acogida y florecer. A diferencia de Olivia Butler, 
para Anzaldúa, la imaginación sí tiene la posibilidad de crear un mundo material nuevo. Al presentar la imaginación como vehículo de la materialización en sus obras, Anzaldúa la utiliza como una estrategia para combatir el trauma de vivir otreado y concebir algo diferente, una realidad nueva con menos dolor para la persona queer.

\section{Bibliografía}

Anzaldúa, Gloria (2009) [1970]: “Reading LP”. En: Keating, AnaLouise (ed.): The Gloria Anzaldúa Reader. Durham: Duke University Press, pp. 250-273.

Anzaldúa, Gloria (1981): “La Prieta”. En: Anzaldúa, Gloria / Moraga, Cherríe (eds.): This Bridge Called My Back: Writings by Radical Women of Color. Berkeley: Third Women Press, pp. 198-209.

Anzaldúa, Gloria (1983) [1982]: “El Paisano is a Bird of Good Omen”. En: Gómez, Alma / Moraga, Cherríe / Romo-Carmona, Mariana (eds.): Cuentos: Stories by Latinas. New York: Kitchen Table Women of Color Press, pp. 153-172.

Anzaldúa, Gloria (1999) [1987]: Borderlands / La Frontera: The New Mestiza. San Francisco: Spinsters / Aunt Lute.

Anzaldúa, Gloria (2002): “(Un)natural Bridges, (Un)safe Spaces”. En: Keating, AnaLouise (ed.): This Bridge We Call Home: Radical Visions for Transformation. London / New York: Routledge, pp. 1-5.

Anzaldúa, Gloria (s.a.): "Speaking in Tongues”. Austin: Gloria Evangelina Anzaldúa Papers / Benson Latin American Collection / University of Texas Libraries, Box 44, folder 19.

Anzaldúa, Gloria / Keating, AnaLouise (eds.) (2000): Interviews / Entrevistas. London / New York: Routledge.

Baldwin, James (1963): The Fire Next Time. New York: Dial Press.

Butler, Judith (1990): Gender Trouble: Feminism and the Subversion of Identity. London / New York: Routledge.

Conner, Randy P. / Hatfield Sparks, David / Sparks, Mariya (1997): Cassell's Encyclopedia of Queer Myth, Symbol and Spirit: Gay, Lesbian, Bisexual, and Transgender Lore. London: Cassell.

Crenshaw, Kimberle (1989): “Demarginalizing the Intersection of Race and Sex: A Black Feminist Critique of Antidiscrimination Doctrine, Feminist Theory and Antiracist Politics". En: University of Chicago Legal Forum 1989.1, pp. 139-167.

Du Bois, W. E. B. (1969) [1920]: Darkwater: Voices from within the Veil. New York: Schocken Books.

Hooks, Bell (1981): Ain't I a Woman. Boston: South End Press.

Hurtado, Aída (2011): “Making Face, Rompiendo Barreras: The Activist Legacy of Gloria E. Anzaldúa”. En: Keating, AnaLouise / González-López, Gloria (eds.): Bridging: How Gloria Anzaldúa's Life and Work Transformed our Own. Austin: University of Texas Press, pp. 49-64.

Keating, AnaLouise (1993): “Myth Smashers, Myth Makers: (Re)Visionary Techniques in the Works of Paula Gunn Allen, Gloria Anzaldúa, and Audre Lorde”. En: Journal of Homosexuality 26.2-3, pp. 73-95. 
Keating, AnaLouise (ed.) (2009): The Gloria Anzaldúa Reader. Durham: Duke University Press. Martin Alcoff, Linda (2006): "Theories and Methodologies - the Unassimilated Theorist".

En: Publications of the Modern Language Association of America 121.1, pp. 255-259. Merriam-Webster Dictionary (2011): “Queer”. En: Merriam-Webster. <http://www.merriamwebster.com/dictionary/queer> (26/04/2019).

Muñoz, José Esteban (1999): Disidentifications: Queers of Color and the Performance of Politics. Minneapolis: University of Minneapolis Press.

Reuman, Ann E. / Anzaldúa, Gloria (2000): “Coming into Play: An Interview with Gloria Anzaldúa”. En: MELUS 25.2, pp. 2-45.

Rich, Adrienne (1980): “Compulsory Heterosexuality and the Lesbian Existence”. En: Signs 5, pp. 631-660.

Sedgwick, Eve Kosofksy (1990): Epistemology of the Closet. Berkeley: University of California Press.

West, Candace / Zimmerman, Don (1987): “Doing Gender”. En: Gender and Society 1.2, pp. 121-151. 
